\title{
El mambeadero, un espace à rituel ouvert chez les Yucuna
}

\section{Laurent Fontaine}

\section{OpenEdition}

1 Journals

Édition électronique

URL : https://journals.openedition.org/clo/2313

DOI : $10.4000 /$ clo.2313

ISSN : 2266-1816

Éditeur

INALCO

\section{Édition imprimée}

Date de publication : 31 décembre 2015

ISBN : 9782858312276

ISSN : 0396-891X

\section{Référence électronique}

Laurent Fontaine, "El mambeadero, un espace à rituel ouvert chez les Yucuna », Cahiers de littérature orale [En ligne], 77-78 | 2015, mis en ligne le 10 mai 2016, consulté le 01 juillet 2021. URL : http:// journals.openedition.org/clo/2313; DOI : https://doi.org/10.4000/clo.2313

\section{(c) (7) (8)}

Cahiers de littérature orale est mis à disposition selon les termes de la Licence Creative Commons Attribution - Pas d'Utilisation Commerciale 4.0 International. 


\title{
El mambeadero, un espace à rituel ouvert chez les Yucuna
}

\author{
Laurent FonTAINE \\ Lacito, Paris 3, Sorbonne Paris Cité
}

Dans toute l'Amazonie du Nord-Ouest, la plupart des Amérindiens sont des mâcheurs de mambe, une fine poudre de coca obtenue après que les feuilles ont été grillées, pilées, filtrées, et mélangées avec des cendres d'autres feuilles (Pourouma cecropiifolia). Du substantif espagnol vernaculaire mambe, est dérivé le verbe mambear ${ }^{1}$ 《 mâcher la coca », et el mambeadero (prononcé aussi mambiadero) : le lieu où l'on mâche en assemblée à proximité du (ou des deux) pilier(s) arrière(s) d'une maloca (grande maison traditionnelle). El mambeadero est un espace de regroupement très particulier, car même s'il s'y déroule toujours des rituels importants, il a surtout pour caractéristique de devoir rendre compréhensible l'essentiel de ses échanges - verbaux et non verbaux - aux non-initiés et aux étrangers (Blancs ou Indiens ne parlant pas les langues de leurs hôtes), contrairement aux autres types de contexte d'origine traditionnelle (situations domestiques, visites, travaux collectifs, cérémonies).

Compte tenu de ce que j'ai déjà dit par ailleurs sur les espaces publics dans la société yucuna (Fontaine, 2013a), el mambeadero apparaît comme un type très particulier de contexte pour deux raisons essentielles. D'une part parce qu'il est crucial pour le changement social. En effet, il offre de nouvelles conditions de communication et d'intercompréhension aussi bien entre les sociétés traditionnelles locales, qu'entre ces dernières et la société englobante ; ce qui permet de le considérer comme un espace public de première importance chez les Yucuna.

1. Beaucoup disent aussi mambiar, du verbe mambear est aussi dérivé un synonyme de mambe : la mambiada (« le produit à mâcher »). 
Et d'autre part parce qu'il est, parmi tous les espaces publics, celui qui est le plus ritualisé du point de vue traditionnel. Nous verrons qu'il est permis d'y observer des pratiques chamaniques relativement complexes, et d'en obtenir non seulement des explications, mais encore les bienfaits attendus.

Beaucoup d'anthropologues ont vu dans el mambeadero le plus haut lieu rituel de la maloca (Serje, 2003 ; Benavides Martinez, 2007 ; Parrado-Rosselli, 2007) : un espace sacré, très chargé symboliquement (Serje, 2003) ${ }^{2}$ où les anciens se réservent le droit de s'asseoir sur leur « siège à penser » (pensador en espagnol) pour mâcher la coca et, par ces moyens, pouvoir y prononcer toutes les paroles considérées comme sérieuses, formelles ou rituelles (mythes, dialogues cérémoniels, répétition des chants, incantations, diagnostics chamaniques, etc.). Ce lieu où l'on mâche la coca dans la maloca apparaît ainsi comme un centre de communication avec les entités surnaturelles (divinités, ancêtres, esprits, auxiliaires chamaniques) permettant d'accroître les pouvoirs d'un soigneur ou chamane dès lors qu'il mâche la coca.

Dans cet article, je ne considérerai pas d'emblée el mambeadero en tant que tel, mais surtout en tant qu'occasion particulière instaurée systématiquement, compte tenu des statuts des personnes qu'elle incite à regrouper, de leurs activités escomptées en un laps de temps donné, et de ce qu'entendent localement les Yucuna par ce terme.

Il faut signaler que le terme mambeadero n'a aucun équivalent dans la langue des Yucuna ${ }^{3}$. D'ailleurs ils ne m'en ont jamais parlé pour s'y référer en tant qu'espace délimité au sein de la maloca. Ce qui n'a rien d'étonnant, puisque l'espace en question peut être employé à différentes fins selon la situation du moment (Jacopin, 1972, p. 117-130) : par exemple, pour étaler des fruits et des tubercules lors de préparatifs de fête (Fontaine, 2001, p. 523-524), des panneaux de feuilles à tresser lors d'un travail coopératif (minga), de la nourriture à offrir lors d'une fête, etc. Concrètement, on peut dire qu'un maître de maloca ouvre et ferme le mambeadero, le temps du moment où il compte y mâcher la coca avec ses invités en plaçant ses sièges sur leurs pieds. Dès qu'il les adosse aux piliers, le temps de penser assis à plusieurs en mâchant la coca est terminé.

Il est également important de signaler que même si les mambeaderos permettent d'organiser des rituels ancestraux mobilisant le plus souvent des compétences

2. Le symbolisme de l'intérieur d'une maloca ayant déjà été décrit et étudié, je n'en ferai pas une nouvelle présentation ici, car cela m'écarterait de mon propos : l'analyse du mambeadero en tant que type de contexte représentatif des espaces publics chez les Yucuna.

3. Il serait intéressant de savoir s'il se traduit dans d'autres langues d'Amazonie du Nord-Ouest. 
chamaniques importantes (divination et prévention des maux), historiquement, ils ne sont apparus que relativement récemment, à la suite des premières assemblées indigènes du milieu des années soixante-dix, comme pour mieux pallier leurs inconvénients du point de vue traditionnel.

Tels que j'ai pu les observer, les mambeaderos se forment à l'occasion des « réunions d'anciens 》 (reuniones de ancianos) également appelées « réunions de nuit » (reuniones de noche) ; ils n'ont de sens et d'intérêt qu'en rapport aux réunions normales (de jour). Les mambeaderos ne sont donc pas à proprement parler des situations d'origine complètement traditionnelle (comme on aurait tendance à nous le faire croire), mais l'un des produits des interactions historiques entre les populations indigènes et la société englobante.

Autrefois, tous les problèmes se discutaient de nuit et en langues indigènes lors des différentes occasions de rencontre traditionnelle (bals, mingas, visites). Quand tout le monde allait se coucher, la seconde partie de la nuit (plus calme et silencieuse) permettait surtout aux soigneurs et chamanes de les traiter avec leurs compétences spécifiques, ce qu'ils considèrent comme beaucoup plus difficile, voire impossible de jour ${ }^{4}$. À partir du moment où les communautés décident non seulement de consacrer des matinées ou des journées entières aux discussions et prises de décision déterminant leurs interactions avec les Blancs, mais encore d'y parler en espagnol pour susciter le dialogue avec leurs représentants, les paroles spécifiques des soigneurs et chamanes se trouvent du même coup exclues de l'ensemble des interventions.

Ceci tend d'un côté à changer les rapports d'autorité au bénéfice de ceux qui connaissent le mieux l'espagnol et le monde des Blancs (les plus diplômés ou acculturés), et d'un autre côté à réduire le rôle du chamanisme dans une société peu adaptée aux transformations sociales radicales ; ce qui ne va pas sans susciter de multiples dysfonctionnements dans son organisation...

Comme le disent souvent les Yucuna à la réunion qui précède une réunion d'anciens : « ce qui requiert le savoir des anciens ne peut pas être discuté ici, mais il sera traité à la prochaine réunion de nuit $»^{5}$.

4. À noter cependant que certaines incantations, en raison de leur longueur, doivent être commencées de jour, et même le matin, pour être arrêtées ou terminées à une heure avancée de la nuit ; par exemple, le baptême du sel végétal (FONTAINe, 2014, p. 123-130). D'autres groupes indigènes préfèrent réaliser leurs soins de jour; par exemple, nous avons observé un chamane Cabiyari réaliser d'importantes cures de jour (ce qui impressionne les Yucuna).

5. Lo que necesita el conocimiento de los ancianos, no se puede tratar aquí, eso se tratará en el próximo mambiadero. 
Du point de vue traditionnel, une réunion de jour apparaît comme un espace de regroupement dont les règles d'organisation ont presque totalement été importées de la société blanche (Fontaine, 2013a). Ces règles vont généralement à l'encontre de celles qui confèrent habituellement aux élites traditionnelles le quasi-monopole des paroles légitimes dans les contextes traditionnels. Malgré les importantes transformations de la société yucuna, les anciens se sont réservé quelques domaines privilégiés où ils ont encore une grande autorité, c'est pourquoi on leur ménage des réunions spéciales de nuit, pour y exercer leurs compétences particulières, mais à condition qu'elles bénéficient à l'ensemble de la communauté et qu'elles soient clairement expliquées à tous.

Dans cet article, je propose d'étudier précisément ce genre particulier de réunion en prenant l'exemple concret d'une réunion nocturne que j'ai pu filmer, pour ensuite en transcrire et traduire les énoncés.

Dans un premier temps, je présenterai le contexte local dans lequel la scène a été filmée, ainsi que le matériel proprement dit.

Dans un deuxième temps, $\mathrm{j}$ 'analyserai les paroles de cette réunion d'anciens en tenant compte du contexte social qui les détermine systématiquement (Jacopin, 1981, 1988, 2008). Selon une méthodologie que j'ai développée (Fontaine, 2001, 2007), je chercherai également à relever leurs conditions contextuelles spécifiques, à caractériser leur ordre séquentiel propre et à expliquer leur symbolisme en les mettant en rapport avec leur cadre de référence.

\section{La communauté de Camaritagua et ses réunions}

Comme la plupart des autres communautés du Bas Caqueta, Camaritagua est une « communauté multiethnique », qui, comme son nom l'indique, est composée de différentes ethnies : principalement yucuna ${ }^{6}$, matapi, miraña, tanimuca et carijona.

Certes, vivre dans une communauté multiethnique a ses avantages quand chacun s'intéresse à la culture des autres groupes pour y trouver une certaine complémentarité. Mais à Camaritagua, cela constitue un défi permanent, car les disputes entre familles d'ethnies différentes sont assez régulières.

6. Par « Yucuna », il faut entendre ici tous les Indiens se disant « Yucuna », notamment en prenant ce terme comme nom de famille. En réalité, sous cette étiquette, il convient de distinguer les «vrais yucuna » (yucuna propio) appelés Kamejeya, des autres groupes ethniques qui en sont venus à parler cette langue, après l'extinction de celle qu'ils parlaient à l'origine, à savoir les Je'rúriwa, les Jimike'pi et les Jurumi. On pourrait dire la même chose des Jupichiya, mais eux ont choisi de se faire appeler Matapi (en tant que nom de famille). 
Presque treize ans après sa création en tant que communauté (en 1990), celle-ci a pu obtenir de l'État, par la résolution n ${ }^{\circ} 12$ du 10 décembre 2002, un territoire propre reconnu comme resguardo (réserve indigène) à l'instar de la plupart des autres communautés indigènes de la zone (resguardo de Puerto Córdoba, Comeyafú, Mirití Paraná, etc.). Cette année-là, elle recense une population de 127 habitants répartis en 26 familles, mais six ans plus tard, en février 2008, cette population a diminué de plus de la moitié, soit 57 habitants pour à peine 13 familles. Que s'est-il passé ?

Pour bien comprendre la situation, il faut savoir que cette communauté a surtout été créée et dirigée par une famille de Miraña, notamment Wasay, un ancien qui en fut le premier capitaine à un âge avancé (plus de 70 ans). De 1996 à fin 2007, il fut relayé par ses trois fils et ses deux gendres (Matapi et Tanimuca). D'un côté, on ne peut nier que les succès et les avantages obtenus au sein de cette communauté reposent en grande partie sur les efforts et l'acharnement en matière de démarches administratives des fils de Wasay (notamment Abraham qui réussit à faire reconnaître le territoire en tant que resguardo). Mais d'un autre côté, les anciens de langue yucuna venus s'installer à Camaritagua (Milciades Yucuna, Mario Matapi et Fermín Yucuna) revendiquent eux aussi leur contribution, précisément parce qu'ils ont largement été sollicités par les Miraña pour « faire avancer les choses » de manière chamanique. Après une longue période d'attente, ce serait de ce point de vue leurs aptitudes chamaniques à influencer les pensées et les décisions des Blancs au moyen de chica ${ }^{7}$ qui auraient été décisives quand l'Institut Colombien de Réforme Agraire (INCORA) accepta finalement de

7. Le chica (yucuna : kena; espagnol vernaculaire : carayurú; nom scientifique : Fridericia chica) est une plante dont les feuilles permettent d'obtenir une peinture rouge. Elle est particulièrement utilisée dans les traitements chamaniques. Aujourd'hui les Yucuna se la procurent généralement auprès des Macuna (sous une forme très sèche et compacte). Généralement, on considère que ce chica permet grâce à certaines incantations de prévoir les problèmes, conflits et dangers liés aux ennemis potentiels, et même d'influencer les pensées, c'est-à-dire de rendre des interlocuteurs plus compréhensifs ou même de changer leur colère en bienveillance (notamment en leur collant discrètement de cette peinture, par exemple, d'une simple poignée de main, ou d'une main sur l'épaule). On dit aussi que cette peinture aide celui qui l'utilise à être plus éloquent et convaincant, c'est pourquoi on s'en met sur la langue et dans le nombril. On peut aussi se dessiner du bout de l'index un trait horizontal sur chaque joue. L'effet est censé durer même lorsque la peinture s'est effacée. Celui pour qui a été réalisée cette protection doit garder sa peinture avec lui (dans une noix spéciale) dans tous ses voyages pour anticiper tout problème. Beaucoup de leaders ou représentants indigènes emmènent donc cette peinture dans leurs bagages pour discuter avec les fonctionnaires et autres responsables blancs à Leticia ou Bogota. 
reconnaître le resguardo de Camaritagua. D'après ce que ces mêmes anciens m'ont dit, ils auraient mobilisé de telles aptitudes à plusieurs reprises dans des réunions nocturnes, les seules assemblées permettant d'organiser des rituels chamaniques au bénéfice de toute une communauté.

Cette discorde, sur la reconnaissance des contributions des uns et des autres, est emblématique de la première des causes récurrentes des tensions à Camaritagua : les luttes de pouvoirs faisant valoir des institutions, connaissances et pratiques divergentes, le plus souvent blanches d'un côté et indigènes de l'autre, mais qui correspondent aussi parfois à des divisions entre ethnies ou groupes de filiation. Ces divergences entraîneront par la suite d'autres désaffiliations parmi les membres de la communauté ${ }^{8}$.

Une autre cause de tension qui fut la plus conséquente jusqu'ici en ce qui concerne les désaffiliations est le refus de se soumettre aux règles instaurées par la communauté et surtout à ses sanctions en cas de transgression? ${ }^{9}$.

Citons celle qui a le plus entraîné de retraits parmi les membres de la communauté : l'obligation de présence et de ponctualité aux réunions ${ }^{10}$.

Cette assiduité est à chaque réunion le premier sujet de tension, puisque l'on y blâme toujours les absents et retardataires. Chacun d'entre eux doit au minimum fournir au capitaine une excuse, et de préférence par écrit (ce qui le plus souvent n'est fait qu'oralement par l'intéressé, ou par un proche durant la réunion). On juge alors de la légitimité de l'excuse, et si celle-ci n'est pas suffisante, on discute déjà de la sanction à encourir (par exemple, une corvée de défrichage des sentiers, ou d'abattage pour la communauté). Beaucoup d'Indiens n'appréciant pas d'être dirigés au nom de la communauté, et ayant de plus l'habitude de se soûler la veille (particulièrement le samedi soir) manquaient évidemment trop souvent aux réunions du lendemain matin ; lassés d'être jugés et sanctionnés systématiquement, ils se sont donc désaffiliés.

8. Parmi ces désaffiliations, il y aura notamment celles de Fermín Yucuna et Mario Matapi.

9. Certains capitaines ne lésinent pas sur les sanctions. Elles sont certes un moyen efficace de « faire respecter les règles ». Mais elles ont également un double désavantage : non seulement elles incitent à la désaffiliation des membres qui se trouvent trop sévèrement dirigés, mais elles sabrent aussi la jovialité, la bonne volonté et la motivation nécessaires à la coopération des membres de toute entreprise collective.

10. Les réunions ordinaires ont normalement lieu une fois par mois, dès huit heures du matin. Pendant longtemps, elles se déroulaient de préférence le dimanche. Depuis que les vols des avions de la compagnie Satena arrivent le dimanche (depuis 2007), elles se font généralement le samedi. 
Enfin, la dernière cause importante de tension est l'insuffisance estimée des bénéfices de l'organisation communautaire (face à ses exigences précédemment mentionnées).

En effet, l'une des attentes essentielles des membres de la communauté tient à la juste répartition des ressources qu'elle obtient auprès de la préfecture de Leticia (Gobernación), notamment grâce à des subventions annuelles (transferencia) distribuées à chaque communauté en fonction du nombre de familles affiliées. À Camaritagua, ces subventions sont principalement distribuées dans quatre secteurs : l'éducation, la santé, l'amélioration de l'habitat et le traitement de l'eau potable.

Ainsi, chaque famille attend avec impatience des tôles de zinc pour couvrir les charpentes et des réservoirs pour le recueil des eaux de pluie. Or certaines familles ont trouvé inacceptable d'avoir à attendre trop longtemps pour ces quelques ressources distribuées en faible quantité. Il faut généralement attendre deux ou trois ans avant de recevoir quatre ou cinq tôles de zinc, ce qui est très peu si l'on tient compte du manque de feuilles de toiture dans la région ${ }^{11}$.

\section{Les réunions d'anciens à Camaritagua}

Toutes les réunions d'anciens que j'ai observées à Camaritagua me sont apparues extrêmement intéressantes, car elles constituent sans aucun doute l'espace indigène où les élites traditionnelles peuvent le mieux faire valoir leur autorité visà-vis d'une assemblée de non-initiés, ce qui offre de nouvelles perspectives pour envisager l'avenir et « résoudre les problèmes ».

Ces réunions sont beaucoup moins fréquentes (deux par an en moyenne) que les réunions diurnes (quinze par an en moyenne) ${ }^{12}$.

Pour avoir un aperçu de l'éventail des activités réalisées lors de ces réunions, je décrirai brièvement les trois réunions nocturnes auxquelles j'ai assisté, avant de présenter plus en détail celle qui fera l'objet principal de cet article.

- Dans la première (le 25 octobre 2004), il était question de régler une menace de malédiction faite par un soigneur (Milciades Yucuna) à l'encontre du capitaine

11. J'ai traité dans un autre article (FonTAINE, 2008) des difficultés à couvrir les charpentes en raison de la rareté des feuilles de toitures (pui en espagnol vernaculaire) due à leur surexploitation.

12. Les réunions diurnes comptent les réunions ordinaires (mensuelles) et extraordinaires. Ces réunions doivent être distinguées des « ateliers » (talleres) qui eux ne réunissent pas toute la communauté, mais uniquement un « comité » nommé généralement pour rédiger un texte administratif ou législatif (par exemple pour la rédaction du statut du resguardo), ou prendre des mesures exécutives. 
Abraham, lors d'un travail communautaire devant un public d'hommes et de femmes venus défricher les sentiers entre les maisonnées. La réunion nocturne mit alors tous les adultes et particulièrement les anciens (au pouvoir chamanique reconnu) face à face pour s'expliquer et voir s'il allait s'ensuivre un emballement des hostilités. Après une altercation entre Milciades et Fermín (de filiation Je'rúriwa) se défiant mutuellement d'engager entre eux une guerre chamanique, Milciades finit par écouter son fils Rey, le priant de présenter ses excuses et de retirer ses menaces. Après cette discussion plutôt animée, Fermín soigna plusieurs personnes de la communauté.

- Dans la seconde (le 15 octobre 2005), tous les incantateurs ${ }^{13}$ de la communauté étaient conviés à faire la prévision chamanique de ce qui allait se passer, et à régler chamaniquement le monde pour éviter les dangers. Cette réunion nocturne était de même type que celle que je vais décrire et analyser en détail un peu plus loin, à la différence que les incantateurs à l'œuvre n'étaient pas exactement les mêmes. On avait notamment mobilisé un chamane (Fermín) pour prononcer l'incantation de la cire d'abeille (mapachara) qui est ensuite brûlée à l'intérieur et autour de la maloca.

- Dans la troisième (le 2 août 2006), alors que la communauté était en train de rédiger le statut juridique de son resguardo à propos du système correctionnel des mineurs, on interrogeait les anciens sur leurs manières traditionnelles de sanctionner, pour juger de la possibilité de les utiliser officiellement sans avoir à faire systématiquement appel à la police de La Pedrera. Malgré des difficultés certaines, la communauté parvint à intégrer partiellement les méthodes traditionnelles dans son système correctionnel (Fontaine, 2008).

Étant donné les informations que j'ai recueillies sur ces réunions, et mes propres observations, je peux dire qu'il s'y déroule principalement deux types d'activités : 1) des discussions sur des thèmes requérants le savoir des anciens et 2) des rituels chamaniques au bénéfice des membres de la communauté.

Ce deuxième type d'activité est précisément celui que je vais présenter maintenant à l'aide de l'extrait de réunion qui va suivre. Cet extrait a été découpé

13. J'emploie le mot «incantateur », comme terme générique pour désigner tous les Indiens exerçant leur chamanisme au moyen d'incantations. J'emploie aussi celui de guérisseur pour désigner les incantateurs qui ont l'habitude de pratiquer des soins thérapeutiques (mais toutes les incantations ne sont pas réalisées dans ce but). Parmi les incantateurs, il faut savoir qu'en langue yucuna on distingue les « soigneurs » (yucuna : lawichú ja'rú; espagnol vernaculaire : curandero) qui uniquement par leurs incantations agissent sur les agents pathogènes, des « chamanes » (yucuna : lawichú; espagnol vernaculaire : brujo) qui pourraient aussi les voir et les extraire. 
au sein d'un enregistrement vidéo de 40 minutes en retraçant les scènes les plus importantes ${ }^{14}$. L'ensemble a été transcrit et traduit une première fois par Edilberto Yucuna, puis une seconde fois par mes soins ${ }^{15}$. Même si plusieurs des dialogues entre anciens sont fort intéressants et auraient mérité d'être étudiés, les limites de place propres à tout article me contraignent à garder ce matériel pour d'autres recherches, et à ne sélectionner que l'extrait le plus pertinent pour l'analyse des spécificités de cette réunion nocturne en tant que type d'espace public. Avant d'en venir au matériel transcrit proprement dit, je vais décrire néanmoins brièvement le déroulement des événements préalables à l'extrait choisi.

\section{Une réunion nocturne chez Mario Matapi}

Le $1^{\text {er }}$ février 2008, tous les adultes de la communauté de Camaritagua étaient conviés à assister à une réunion d'anciens dans la maloca de Mario Matapi, à la fois maître de maloca et soigneur reconnu. Il était question de faire des incantations, d'une part pour « manier le monde » (eja'wá maná la'kaje) ${ }^{16}$, c'est-à-dire prévoir, esquiver ou réduire les risques de « maux » (chapú nákaje) susceptibles d'atteindre les membres de la communauté et, d'autre part, pour savoir quand il serait possible de clore les deuils qui attristaient les familles de Milciades et de Fermín $^{17}$. Par conséquent, on comptait essentiellement en ce genre d'occasion sur

14. L'intégralité de cette vidéo est en ligne sur mon site internet (FonTAIne, 2015a), ainsi qu'une version courte de 20 minutes. Les sous-titres peuvent notamment être visionnés en français et en yucuna.

15. Ce type de travail étant long et compliqué, Edilberto est parfois assez sommaire dans ses transcriptions et traductions. Même s'il est indispensable pour éviter les malentendus et erreurs de traductions, ce travail du traducteur indigène n'est finalement qu'un premier dégrossissage. Je m'en aperçois particulièrement lorsque je cherche à « coller au plus près » de la vidéo en y insérant des sous-titres. Il me reste alors à combler les phrases manquantes ou trop succinctes par rapport à ce qui est véritablement saisissable dans l'enregistrement.

16. «Le maniement du monde » est le titre de l'ouvrage de Maria Clara Van der Hammen sur les Yucuna (El manejo del mundo, 1991). L'incantation employée eja'wá maná la'kaje peut se traduire par « invocation du monde ».

17. Milciades venait de perdre deux de ses petits-enfants : la fille de sa fille cadette, âgée de 5 ans, morte noyée 10 mois plus tôt, et le fils de sa fille aînée, âgé de 19 ans, qui venait de se pendre 15 jours auparavant, après une beuverie dans le village de La Pedrera (j'y reviendrai). L'un des frères cadets de Fermín venait de perdre ses deux jeunes enfants, noyés dans les rapides de Córdoba. 
la présence des «anciens » (yucuna : pheñáwani; espagnol : ancianos), c'est-àdire les soigneurs et chamanes maîtrisant les rituels à l'ordre du jour de la réunion.

Comme à chaque réunion nocturne, le rendez-vous avait été fixé à 18 heures, afin que les participants aient la possibilité d'arriver juste avant la tombée de la nuit ${ }^{18}$. Ceux-ci s'assoient alors en différents petits groupes à l'intérieur de la maloca.

Tout d'abord, Milciades Yucuna et Mario Matapi, les deux principaux anciens sur lesquels on compte pour les rituels, s'assoient pour converser sur des sièges traditionnels à proximité du pilier avant gauche de la maloca.

Pendant ce temps, d'autres adultes vont utiliser les autres sièges à proximité du pilier arrière gauche pour dialoguer et plaisanter. Parmi eux, il y a les deux fils de Milciades : Rey Yucuna, qui vient seulement d'être nommé capitaine deux semaines plus tôt, et qui sera notamment chargé de traduire en espagnol ce que les anciens aviseront publiquement une fois leurs incantations terminées; et son cadet Edilberto Yucuna, également chargé de faire l'incantation de « maniement du monde » avec de la cire d'abeille. N'étant encore qu'un jeune soigneur que son père s'apprête à déclarer publiquement comme tel après la clôture du deuil, il ne fera ici qu'une partie de l'incantation qui sera reprise ensuite par Mario. Ceci permet à Edilberto de mettre à l'épreuve ses compétences devant un autre soigneur que son père, pour être ainsi mieux reconnu.

Enfin, un autre soigneur est venu s'asseoir pour participer activement au rituel : il s'agit de Káiwa Miraña, l'un des deux seuls anciens - avec Wasay Miraña - de la communauté à parler la langue miraña et à pratiquer le chamanisme. Notons toutefois que le chamanisme de ces deux Miraña est beaucoup moins valorisé que celui de Milciades et Mario ${ }^{19}$.

Quand arrivent en bloc les autres adultes de la communauté, les deux soigneurs principaux amènent leurs sièges à proximité du pilier arrière gauche auprès des autres adultes précédemment mentionnés. Rey laisse les quatre soigneurs ensemble, et organise la disposition du lieu pour recevoir les nouveaux arrivants.

18. La région de La Pedrera se trouve en plein sur la ligne de l'équateur. Par conséquent, toute l'année, le soleil se couche aux environ de 18 heures (et se lève vers 6 heures).

19. S'il est moins reconnu, ce n'est pas parce que le chamanisme des Miraña est moins valorisé que celui des Yucuna. Leur faible reconnaissance tient à trois raisons essentielles : 1) l'origine de leur formation chamanique reste floue, c'est-à-dire qu'elle ne s'appuie pas sur la renommée d'un soigneur reconnu ;2) ils auraient abandonné leur milieu traditionnel miraña durant leur adolescence, par conséquent ils n'ont pas reçu de formation aussi longue et approfondie que Milciades et Mario ; et 3) ceux-ci n'ont jamais pu prétendre soigner autant de maux que ces derniers. 
Après avoir laissé les quatre bancs traditionnels aux soigneurs, il ne garde pour lui qu'un simple petit siège (généralement utilisé par les femmes pour râper le manioc) et place les deux longs bancs de la table ${ }^{20}$ de Mario face à lui, entre les deux piliers avant de la maloca, pour pouvoir s'adresser à eux. S'y installent alors tous les autres hommes importants de la communauté (notamment Wasay et ses fils).

Quant aux femmes, elles sont restées en retrait sur les bancs de la périphérie. Derrière ces bancs, certaines ont déjà installé leur hamac et s'y sont même allongées avec leurs enfants.

Vers 18 h 30, quand tous les membres de la communauté sont réunis, Mario offre un rapide repas de bienvenue en mettant à disposition au milieu de la maloca des galettes de cassave et de la sauce de manioc ${ }^{21}$.

Ensuite, tous les anciens placent au milieu de la maloca le pot $^{22}$ de coca qu' ils ont personnellement apporté en l'occasion de ce mambeadero. Les hommes viennent alors se servir un peu de chacun des pots présentés ${ }^{23}$.

Vers 19 heures, la première étape de la réunion durant laquelle étaient attendus tous les adultes de la communauté est terminée.

Ceux qui ont apporté des cigarettes se chargent d'en offrir particulièrement aux quatre anciens, et devront s'assurer qu' ils en auront suffisamment tout au long du rituel.

À nouveau assis, les quatre anciens se partagent les différentes incantations. Milciades demande à Mario son porte-calebasse (umichiripuku) pour y déposer la petite calebasse remplie de la coca qu' il va traiter chamaniquement. Comme Mario

20. Mario a dans sa maloca une grande table de 2,5 mètres sur 0,80 avec deux bancs que lui a offerts un Brésilien, longtemps resté fiancé à l'une de ses filles.

21. Wa'lako (yucuna). Tucupi (espagnol vernaculaire). Il s'agit de jus de manioc amer cuit assez longtemps jusqu'à obtention d'une sauce marron foncée, souvent mélangée avec des piments. Elle sert ordinairement à relever le goût de la cassave, surtout lorsqu'il n'y a rien d'autre à offrir (ni viande ni poisson).

22. Aujourd'hui, on utilise des boîtes de lait ou de chocolat en poudre, c'est-à-dire des boîtes en métal ou en plastique pouvant se refermer hermétiquement avec un couvercle. Autrefois on employait des calebasses évidées.

23. On utilise au mieux une cuillère traditionnelle spéciale appelée ta’pá, creusée dans un fémur de tapir, sinon une grande cuillère métallique à laquelle on a cassé le manche (pour rentrer plus aisément dans la boîte lorsqu'elle est pleine). Faute de cuillère, on peut aussi utiliser une simple feuille (parfois on arrache un morceau de feuille de toiture). Dans d'autres occasions moins formelles, et surtout si la boîte à une ouverture étroite, on fait comme si on buvait directement la poudre de coca à même le pot. 
n'a pas d'autre umichiripuku, tous les autres vont se contenter de garder dans leur main l'objet qu'ils vont traiter. Káiwa commence à se pencher sur sa calebasse de coca en marmonnant silencieusement des paroles que lui seul semble comprendre. Mario prête ensuite son morceau de cire d'abeille à Edilberto, et prend la calebasse de genipa qu'il avait préparée pour se pencher dessus également. S'ensuit alors un long moment (d'une heure et demie à deux heures) durant lequel, chacun des quatre hommes est penché sur son objet chamanique en murmurant sa propre incantation. Au début de l'incantation, puis à plusieurs reprises, chacun d'entre eux souffle de la fumée de tabac sur son objet. Avant d'en venir à l'annonce de ce qu'il a ressenti, Milciades parle cérémoniellement avec Mario.

\section{Extrait de transcription séquentielle de la réunion nocturne chez Mario Matapi}

1. Túwemi i'maká rili'chaje lawichú yukuna Makolakamijlo.

\section{Milciades informe Rey sur le traitement chamanique qu'il vient de faire avec de la coca. *}

2. Milciades

Palá nuli'chaka riká i'maká.

3.

4.

5.
E’iyonaja kaphí kuwani kariwana nakuwa i'michaka nojló.

Marí kaje aú nulapachiya nomákaloje apala nala’a weká chapú ka’jná.

Palani ka’jná nu’rí.
Ça s'est bien passé.

Mais j'ai eu la forte sensation d'une arrivée de Blancs.

Alors j'ai énuméré toutes les éventualités pour voir s'ils allaient nous faire du mal.

Mais ça devrait aller, mon fils.

\footnotetext{
* On remarque que les descriptions en italique sont transcrites également en yucuna. Comme pour les énoncés, il s'agit des propres phrases du traducteur Edilberto Yucuna. J'ai tenu à les reproduire intégralement pour mieux tenir compte de ses propres informations, plutôt que de les changer pour en apporter d'autres qui n'auraient pas forcément été plus pertinentes. Si d'autres informations sont nécessaires, je les indiquerai à part en notes ou dans mes propres commentaires. On pourra également se reporter à la vidéo sous-titrée (FonTAINE, 2015a).
} 
6. $\begin{array}{ll}\text { Nulapachiya : Je'rúriwa } & \text { J'ai nommé les différentes } \\ \text { kaje maka, Jimike'pi ka'jná, } & \text { tribus : Je'rúriwa, Jimike’pi, } \\ \text { Jurumi kaje maka... } & \text { Jurumi, etc. }\end{array}$

7. Palani nu'michaka ñakeji Et je n'ai ressenti aucun nakú. signe inquiétant.

8.

Pimá najló mete'ni : "Unká na i’malá."

Maintenant tu vas leur dire qu'il n'y a rien de spécial.

9. Rey

Jm jm.

Bien.

10. Milciades

Kaja piyuke nuli'chakajla riká.

J'ai déjà tenté d'aborder tous les types de maux.

11. Kaja nutajnáchiyajla riká piyuke ina'uké chi' ná keño'ko ejena.

12.

Pumí chiyá nukeño'cha merañana. Palani. E'iyonaja nayajalo numá chiyá kaphí kuwani chapujeji i'michaka nojló.
Et j'ai terminé de mentionner toutes les catégories humaines avec leurs terres d'origine.

Seulement avec les Miraña, j'ai clairement ressenti que de mauvaises paroles allaient jaillir de la bouche de leurs femmes.

13. E Makolakami i'má Rey s'apprête à traduire cela aux riyukuna ina'ukejlo. personnes rassemblées.

14. Rey Owilá !

Mon oncle !

15. Julian

Ee !

Oui !

16. Rey

Kaja.

Ça y est.

17.

Solín, pajñá pikakuwa. Solín, mâche ta coca. 
18.

19. Mario

20. Rey

21.

22.

23.

25.

26.

27.

28.

29.
E ta marí nakaje to'ro Et ça en dessous ? ja'pá ?

Keja ri'micha.

C'était là.

Aa! Maere ri'michá richu. Ah bon ! C'était à

l'intérieur.

iAbraham!

Abraham !

Aqui mi papa hizo una

Là, mon père a fait une cure curación para nosotros. pour nous.

Para mirar cómo vamos a Pour voir comment nous estar trabajando juntos. allons travailler ensemble.

No hay ningún problema. Il n'y a aucun problème.

Entonces, para ustedes, Mais pour vous et vos para las mujeres de ustedes, femmes, attention, s'il vous porfavor hay que tener en plait, à ne pas susciter de cuentaque no van a meter problèmes. problemas.

Cualquier cosa que ustedes miren.

Pour quoi que ce soit qui vous déplait.

Eso si le señalo muy duro Ça, il a fortement ressenti qu'il pouvait y avoir des problèmes.

haber problemas.

Entonces para evitar eso pues, hay que retener eso.

Donc pour les éviter, il faut retenir cela. Antes que se agranda Avant que cela ne s'aggrave, mucho, hay que frenar eso. il faut le freiner. 
30.

31.

32.

33.

34.

35.

36.

37.

38. Un

Tanimuca

nouvellement

affilié à

Camaritagua
Entonces de problema, no Sinon, il n'y a pas de hay ningún problema. problème.

De pronto hay un problema por allá, pero para nosotros aqui, no hay ningún problema.

Bueno, en cuestión de los Pour des questions de Blancos pues, ellos allá... Pero para nosotros no hay ningún problema con ellos.

Il peut y en avoir un, là-bas, mais pas chez nous.

Blancs, ça les regarde là-bas... Mais pas pour nous.

Pero si van a estar

Néanmoins ils vont

llegando, como siempre les rencontrer, comme ellos que llegan allá.

d'habitude, ce sont eux qui vont là-bas.

Surtout les militaires... militares...

Eso es lo único que le C'est la seule chose dont il a ressenti un mauvais signe. señaló mal.

Donc ça, c'est pour vous en Entonces eso para que mettre sur le corps. echen alli en el cuerpo.

Mon non-parent, tu peux te mettre de la coca. ipatú.

Bien ! 
39. Rey

40.

41. Un jeune

42. Hernán Miraña

43. Rey

44.

45.

46.

47. Wasay

48. Rey
Nuteló Juni Jalomi.

Mere chi inaana ?

Kele inaana.

Yee ! Pajñá !

O’weló Olinda !

Piji'cha pinakuwa kaja!

"Unká inanaru !" dijó

Wasay.

Eso es para todos.

Llenó mi bota todo!

Eso es una defensa

personal.
Non-parent, Mère de l'Eau.

Où est la dame?

La voilà.

Oh ! Fais-toi-le donc !*

Grande sœur Olinda !

Prends-en aussi.

« Pas une demoiselle ! » a dit Wasay.

C'est pour tout le monde.

Ma botte en est entièrement remplie !

C'est une défense

personnelle.

49. Lana aú Mario la'á ri'maká. Après avoir fait sa cure avec du genipa, Rejomi ra'ó piño rile'jé Mario informe à son tour (devant yukuna i'maje. Milciades) de ce qu'il a perçu. **

\footnotetext{
* Pajñá ! Littéralement « Mange! ». Il s'agit d'une plaisanterie fréquente entre hommes (trois au minimum) dès que la situation le permet (même brièvement comme ici), et que tout le monde sait dire à Camaritagua (même les Miraña). Dès lors que l'un des hommes parle de quelque chose qui peut sous-entendre une invitation sexuelle à un second, un troisième larron ne manquera pas de dire cela au second. Cette plaisanterie détend l'atmosphère en suscitant toujours le rire collectif dans les communautés proches de La Pedrera. Mais les autres indigènes de langue yucuna venus du Mirití s'indignent souvent devant cette transgression des règles de respect, surtout quand les relations de parenté sont proches.

** Edilberto oublie de mentionner que Mario vient aussi d'échanger des paroles cérémonielles avec Milciades comme préalable à son annonce.
} 
50 Mario

51.

52.

53.

54.

55.

56.

57.

58.

59.

60.

61.

62.

63.
Marí nuli'chá wajló

lawichú.

Nomakaloje meke we'jichá rijwa'té.

Pala nuwe'picha.
J'ai aussi fait du chamanisme pour toutes les personnes présentes.

Pour voir comment ça va aller.

Mes sensations ont été bonnes.

E’iyonaja nupechu nakú... Cependant certaines ont été mauvaises...

C'est l'impression que m'a

laissée la coca.

Marí ke ri'micha nukaku ja’pejé.

Nuwe’icha mujrí nakú. J J ai ressenti la grippe.

Aa ! Jeí nakú nuqueño’o

Ah oui! J'ai commencé par i’michá. les morsures de serpent.

Jeí nulapachiya piyuke

J'ai énuméré tout le monde.

Kamejeya,

Les Yucuna,

Wekó,

Ma tribu matapi,

Merañana,

Les Miraña,

Je’rúriwa,

Les Je'rúriwa,

Pe'iyojona.

Les Tanimuca.

Ke’iyapé weká re kele jei ke neí pe'iyojona weiya

Pour ces derniers qui vivent parmi nous, j'ai ressenti quelque chose. 


\section{Milciades $\mathrm{Hmm}$ ?}

65. Mario

66.

67.

68.

69.

70.

71.

72.

73.

74.

75.

76.

77.

78. re’iyá.
Pe’iyojona kajena

Kanakuwa i'michá neí nakú,

Upejlú riká kaja pe'iyojona weiya i'makana

Inaana kamejeya mená e'iyajé...

Upejlu ne'micha mená

Marí ri’micha nojló.

Eyá nuji'cha mujrí.

Marí mujrí waicha.

Iyama ke pe riwaicha kewini waní!

Unká meke i'malá

E yuwaná, nuká ta, richa'ta.
Pour qui ?

Les Tanimuca.

Leur nom m'en a donné la sensation.

Attention à celles qui vivent avec nous.

Les femmes yucuna dans les jardins...

Qu'elles fassent attention.

C'est ce que j'ai senti.

Puis j’ai ressenti la grippe.

Elle revient.

Elle va frapper deux fois, et très fort.

On n'y peut rien.

Elle va gagner les enfants, et même moi.

Pala namaje yuwaná mujrí Il faudra faire attention à celle des enfants.

Yuwaná, unká chutaka, Ne pas rester à côté d'eux yechi ne'makaje i'maka. quand elle va commencer...

eyá pheñawila ñaají ke. Sinon les adultes aussi vont l'attraper. 
79.

80.

81.

82.

83.

84.

85. Mario à quelqu'un s'apprêtant à l'interrompre

86. Mario

87.

88.

89.
Eya nuji'cha ji'wákajiwa. Puis j'ai nommé le paludisme.

Ilé kaje pala, unká na Ça va, rien à signaler. i'malá

Eya nukeño'cho aphala Ensuite, j'ai mentionné les jaruna, piyutena, jarechina, la'yana, jémana. fourmiliers, les anacondas, les esprits telluriques, les tapirs.

J'ai ressenti la marque des tapirs.

i'micha.

Il faudra en parler aux enfants.

kanakuwa i'micha.

Pour les cerfs...

Kawayana...

Attends !

¡Espere!

Attends !

Kele kawayana, ñakeja. Pour les cerfs, c'est pareil.

Palani ka’jná.

Eya nu'majla walijipuku. Attention à leur viande récemment fumée.

Walijimana, yuwana, iyani Pour les adolescents, les yami ka'jná, enfants, les parents qui viennent d'avoir un enfant...

walijipuku kaje ka'jná, précaution.

makajwata, 
52 Paroles publiques, paroles confidentielles $-\mathrm{n}^{\circ}$ 77-78

90.

Riká i'michari nojló J'ai senti que si on en pajñaka chu kanakuwa, mange, le tonnerre peut kechami pichani i'micha frapper. ñakeji nakú.

91.

Riká i'michari nojló. C'est ce que j'ai senti.

Unká meke nu'malá. Je ne peux pas m'y opposer.

93.

Pala nema'ka.

Ils ont déjà entendu [mes avertissements].

94.

Naké numá najló nakaje : Je leur avais dit :

95.

96.

Wajé nakaje keño'jiko,

Dès le début, quand leur kaja jupimi ne'makana capitaine en parlait... kemá nakú...

97.

La'jeri kele lawichú, pa Ils avaient pris ce chamane, a’jná et puis...

Wajé na'je natamí ka'jná, Ils étaient encore plus unká chu rutejmo'lo. malades, et elle ne guérissait pas.

98.

E pala la'kaño

Ils doivent bien la faire lawichojona! soigner!

99.

Eya kaja nu' micha Je suis allé chez eux, najwa' té meyale. récemment.

100.

— Meke pi'micha?

- Qu'as-tu senti ? [m'at-on demandé]

101. Me pacha jema kanakuwa kemachi nojló !

J'ai ressenti que la faute envers les tapirs est grande ! 
102.

103.

104.

105.

106.

107.

108.

109.

110. Milciades Na ta riká ?

111. Mario

112.

Karíwana.
Eya ka'jná kele okuru phíyu'ke kele kanakuwa chiya nakaje kají nakaje i'micha nojló.

Eya numaji chuwa ruyani ka’jná : "Pikupaji kele jaló...

La'kajna we' pi pala rojló lawichokana, lana la'kana rojló."

Eta pala rojló nuwe'icha.

Ñake marichú la'jika rojló.

Maareya nu'jicha wapechuwa nomakaloje meke we'maje.

Ilé kariwana, meke i'majika?

Kariwana kanakuwa keño'ko iphaka, jácho’o panakuwaka.
Et c'est ma pauvre vieille tante qui en fait les frais.

Alors j'ai dit à ses enfants : «Si vous ne voulez pas perdre votre mère...

Prenez-lui quelqu'un qui soigne bien avec du genipa. »

Là j'ai senti qu' elle irait mieux.

C'est cela que doit faire le chamane.

De là, j'ai continué à suivre notre pensée...

Quant à ce qui va se passer avec les Blancs...

J'ai senti que d'autres Blancs [la guérilla des FARC] allaient surgir, pour les affronter.

Affronter qui ?

Les Blancs [militaires].

Mais cela ne se passera pas Maareya ta ka'jná ?
ilé karíwana waicha. ici. 
54 Paroles publiques, paroles confidentielles $-n^{\circ}$ 77-78

113.

114.

115.

116.

117.

118.

119.

120.

121.

122.

$$
\begin{aligned}
& \text { Unká ilé ño'jó na'pala. } \quad \begin{array}{l}
\text { Comme ils marchent en } \\
\text { Eja'wá e'iyá na'paka, eya forêt, ça se passera là-bas. } \\
\text { na'paje re i'majeri. }
\end{array}
\end{aligned}
$$

Unká kaje i'mala. Riká J'ai senti trois coups très wejí ke pe weichaka nojló, forts.

kaphí nuphá.

Pácho'jo iñe'pú chuwá pueblo ejó.

Alors ils vont rentrer au village.

Rikaja kalé.

C'est tout.

Eya yéwicha wapechu Puis je me suis recentré sur nomá meke we'maje. nous.

Marí ke we'majika Sur ce que nous allons faire. me'teni.

Marí ke nu'má me'teni Comment va se dérouler jarechi, kajmú atapi, pipirí cette fin d'été. atapi.

Palani, we'majla wale'jé, Ça va. Nous pourrons faire we'rakaloje mawirú ka’jná. notre propre bal et boire l'alcool d'ananas.

Walama'a wale'je wai, Préparer nos ananas quand wale'je mawirú, keraphí ils seront bien mûrs. riká ka’jná.

Keño'ko ilé lukamá $\quad$ Est-ce que ce sera au début ja'pejé, keño'ko lerú de l'hiver ? À l'arrivée de awa'á we'jicha, apa ka'jná l'été ? ri'maji wajló. 
123.

124.

125.

126.

127.

128.

129.

130.

131.

132.

133.

134.
Mawirú, keraphí mawirú Quand les ananas seront ka’jná. rouges.

Mawirú jarechi ka'jná. Probablement lors de l'été des ananas.

Nulapachiya kele jarechí. J'ai mentionné cet été.

Unká, palá ke numicha. Pas de problème.

E iyamata keri. Le second mois...

Kaapukú aphata keri. À̀ la pleine lune, après un mois.

Ilé no'jó nupha pala nakú À ce moment-là, il sera bon kele keri chapú nu'má de préparer la fin du deuil. la'kana.

Eya marichú numaje nakú J'en avais parlé à Fermín, le nakaje. chamane.

Eya kele marichú iphajika, Il s'en occupera en rentrant. mekelana chapú i’maká...

Eya riká, “nula’je «Je préparerai la fin du rikapi' íchata” ke rimicha. deuil » a-t-il dit.

Nake riphajika ee wala'je Alors nous attendrons son rinakoje, wamaje meke retour.

ri'má riká.

Kaja numicha ijló marí ke Donc je dis que ce sera plus ñaapa, aphata kaapukú. tard à la pleine lune du mois prochain.

Aphata kaapukú, ejená Il vaut mieux attendre nojló palá. jusque-là. 
56 Paroles publiques, paroles confidentielles $-\mathrm{n}^{\circ}$ 77-78

135.

136.

137.

138. Rey à l'assemblée

139. Rey à Hernán

140. Hernán

141. Rey $\dot{a}$ l'assemblée

142.

143.

144.

145.

146.
Eyá meji'wakaje wajló pheñawila ta, kiñaja, iná we'piri i'majlá.

Kele meji'wakaje wajló, unká i'malá ta. Kaphí ta unká i'malá.

Marí ketanaja nuwe'ichaka wajló.

Aqui hay otro.

Pajñá oiga!

Pajñá oiga!

Aqui está el vaso.

Este es otro.

Eso es también una defensa, pero otro.

Ahorra ellos pensaron como Ils viennent de penser podrian arreglar el tiempo. à comment ils allaient arranger le mauvais temps.

Entonces, para dos meses, Donc, ce sera bien pour dans un mes y medio, está bien.

De alli para adelante, está un poco mal.
Quant à l'exposition aux autres maux, même rapidement on peut savoir.

Selon moi, il n'y aura rien d'autre.

C'est tout ce que j'ai appris pour nous.

En voici un autre.

Fais-toi le je te dis !

Mange-le toi-même!

Le bol est là.

C'en est un autre.

C'est aussi une défense, mais différente.
Après cela, ça n'ira plus très bien. 
147.

148.

149.

150

151.

152.

153.

154.

155.

156.
Entonces, de aqui dentro de un mes y media, ellos van a comenzar a arreglar el tiempo...

para comenzar a organizar pour commencer à préparer para un baile.

Entoncespara los Miraña Pour les Miraña qui ont des que tienen mujeres yucuna hay que cuidarse mucho porque

la culebra esta peligrosa para ellas.

Eso hay que cuidar muy bien.

O los Yucuna, no solamente mujeres, para nosotros también hay.

Eso es lo único que le señaló mal.

¿Entonces hay que tener mucho cuidado!

$Y$ en cuestión de mi abuela, hay que hacerle un tratamiento tradicional porque ya está muy...

Como que la danta quiere llevarla será.
Dans un mois et demi, ils vont arranger les conditions atmosphériques... un bal. femmes yucuna, attention parce que...

les serpents sont dangereux pour elles.

Il faut y faire très attention.

Pour nous les Yucuna également, pas seulement les femmes...

C'est la seule chose dont il a ressenti un mauvais signe.

Donc attention!

Et pour ce qui concerne ma grand-mère, il faut lui faire un traitement traditionnel parce qu'elle est très...

On dirait que le tapir veut l'emporter. 
58 Paroles publiques, paroles confidentielles $-n^{\circ}$ 77-78

157.

158.

159.

160.

161.

162.

163.

164.

165.

166.

167.

168.
Entonces, hay que llevarla Il faut donc l'amener à un a un tradicional que sabe ancien qui sait là-dessus. eso.

Para que le haga una defensa, y eso lo va hacer con esta misma pintura.

Que asi ella pueda estar más tiempo ya.

Eso no más.

$Y$ entonces pues, para los Blancos si, como siempre ellos...

Van a llegar, pero por acá no hay problema con nosotros.

¿Eso ya ellos allá, no?

Pero nosotros no buscamos problemas con ellos.

Eso fue todo.

Entonces esto, para que se unten.

Aqui o en el pie lo que sea.

Aqui o en el corazón, eso no más.
Un peu ici ou sur le pied

Pour qu'il lui fasse une défense avec cette même teinture.

Pour qu'elle puisse vivre plus longtemps.

C'est tout.

En ce qui concerne les Blancs, comme toujours...

Ils vont les rencontrer, mais comme ici il n'y a pas de problème avec nous.

Ça se passera avec eux là-bas, n'est-ce pas ?

Nous, nous ne cherchons pas à avoir de problèmes avec eux.

Et c'est tout.

Donc voilà, mettez-vous-en. Ici ou sur le cœur, et c'est tout. 
169.

170.

171.

172.

173. Milciades

174. Rey

175. Mario

176. Rey

177.

178.

179.
Mire, esto es...

Los que tienen torcido por alli hay que tener mucho cuidado porque eso si esta ...

¡El que se va hacer pillar.. allá!

O’weló Olinda ! Pajñá apú piño!

¡Y tener mucho cuidado cuando van a tomar!

$\cdots$

Pajluwata keri apú nakoje?

A'a. Riwakaje.

Re jo’o wawakajiko apú piño ilé ño'jó.

El viejo me dijo que dentro de un mes vamos a reunirnos para hacer mambiadero otra vez.
Regardez, ça c'est...

Ceux qui ont fait des coups tordus dans le coin, attention!

Celui qui va se faire prendre... là-bas [en prison] !

Grande sœur Olinda ! Viens mâcher à nouveau.

[inaudible]

Et attention quand vous allez boire !

[inaudible]

Le mois prochain ?

Oui. À ce moment-là.

Il faudra encore nous réunir ici.

Le vieux vient de me dire que dans un mois, on se réunira pour faire un mambiadero à nouveau.

Ensuite, c'est au tour d'Edilberto d'échanger des paroles cérémonielles avec Mario, puis de lui annoncer ce qu'il a ressenti lors de son incantation. 
CAHIERS DE LITTÉRATURE ORALE

60 Paroles publiques, paroles confidentielles $-\mathrm{n}^{\circ} 77-78$

Ceci sera également traduit à la communauté par Rey ${ }^{24}$.

\section{Les conditions récurrentes des réunions nocturnes}

Tout d'abord, ce qui permet de qualifier une réunion nocturne en tant qu'assemblée est qu'elle partage les mêmes règles générales que les autres assemblées ${ }^{25}$.

Mais elle est un genre particulier dans la mesure où nous trouvons également des règles particulières. La première est :

1) L'obligation de mobiliser le savoir et les paroles chamaniques des anciens. Cette règle est ici principalement appliquée lors des incantations murmurées (n'ayant pu être transcrites) et des annonces des résultats des incantations (séquences 2-8, 10-12, 50-63, 65-84, 86-109, 111-137, 173-177).

Or cette règle particulière apposée aux règles générales requiert une seconde règle particulière qui permet d'éviter la transgression de certaines règles générales.

Comme le savoir et les paroles chamaniques ne sont pas directement compréhensibles par tous, et que dans toute assemblée règne « l'obligation pour tous de comprendre, et donc le droit pour tous de comprendre $»^{26}$, une autre règle doit nécessairement être créée pour assurer le respect de ces deux dernières règles :

2) L'obligation de traduire et d'expliquer les paroles chamaniques des anciens. Ce sont ainsi les applications de ces deux règles qui permettent à coup sûr de reconnaître une réunion nocturne en présentant à la fois des énonciations de type chamanique et des traductions en espagnol de ces énonciations devant l'assemblée. La coprésence de ces deux types de paroles est absolument fondamentale pour la caractériser. Si elle ne présentait que des énonciations de type chamanique, il pourrait s'agir de n'importe quelle veillée indigène non communautaire et non publique. Et si elle ne présentait que des traductions de paroles non chamaniques, elle ne se distinguerait pas de tout autre genre d'assemblée dans laquelle on parle plusieurs langues.

24. La suite a été filmée durant un quart d'heure puis enregistrée au magnétophone jusqu’à la fin de la réunion. Malheureusement, la place manque ici pour étudier la suite de l'extrait.

25. Ces règles sont : 1) l'obligation d'annonce, 2) l'obligation de présence, 3) l'obligation de direction de l'assemblée, 4) l'obligation de finalité de l'information, 5.a) l'obligation pour tous de comprendre, 5.b) le droit pour tous de comprendre, 6) l'interdiction de perturbation, 7) le droit de parole devant l'assemblée, 8) le droit de diffusion extérieure, et 9) le droit d'expression et de critique à propos de l'assemblée (Fontaine, 2013a, p. 92-95).

26. Laurent Fontaine, 2013a, p. 93. 
De nombreuses séquences supposent le respect de ces deux types de règles caractéristiques des réunions d'anciens.

- Dans la séquence 8, Milciades dit à son fils : « Maintenant tu vas leur dire qu'il n’y a rien de spécial ». Il parle de ce qu'il a ressenti au cours de son incantation et demande à son fils de traduire ses paroles aux membres de la communauté.

- Dans la séquence 13, la description d'Edilberto explique que Rey va procéder à la traduction de l'annonce chamanique de son père.

- Dans les séquences 22 à 35, Rey traduit en espagnol à Abraham et aux autres membres de la communauté, ce que son père lui a dit de ses impressions chamaniques.

- Dans les séquences 36, 37, 44, 46, Rey traduit toujours en espagnol ce qu'il connaît de la procédure de protection chamanique avec de la coca. Il incite l'assemblée à se mettre de la coca sur le corps.

- Dans les séquences 138 et 141 à 165, Rey traduit cette fois devant l'assemblée l'annonce chamanique de Mario.

- Dans les séquences 166 à 168, il demande à l'assemblée de se mettre du genipa sur le corps.

Mis à part ces actes appliquant des règles propres aux réunions d'anciens, je dirais qu'il n'y a rien d'étonnant à trouver des genres d'énonciations présents dans d'autres occasions, mais néanmoins obligatoires dans ces réunions d'anciens : il s'agit des paroles cérémonielles et des plaisanteries que l'on retrouve notamment dans tout rassemblement cérémoniel (Fontaine, 2013a, p. 85).

De même que dans toute autre assemblée, on rencontre également de la part du capitaine Rey des appels d'attention vis-à-vis de certaines personnes ou de l'assemblée en général (séquences 14, 21, 43, 138), ou encore des appels à participation dans le rituel (séquences 17, 37, 39, 43-44, 46, 166-168, 172). Pour le capitaine, ces actes sont indispensables à l'application de la troisième règle générale à toute assemblée : l'obligation de direction.

\section{Le déroulement des réunions nocturnes}

Dans toutes les réunions d'anciens que j'ai observées, on retrouve toujours un même ordre de base général pouvant être précisé en fonction des différents types d'activités du jour.

Dans le tableau suivant, je présente l'enchaînement récurrent des actes selon leur ordre de base (première colonne), et indique les variations possibles : en l'occurrence, les actes 5 et 6 (le partage de la coca et des cigarettes) peuvent être optionnellement adjoints à d'autres moments que l'ordre de base (seconde colonne). 


\begin{tabular}{|c|c|}
\hline Enchaînement fondamental des actes & $\begin{array}{l}\text { Adjonctions facultatives } \\
\text { de certains actes }\end{array}$ \\
\hline 1) Arrivée des invités & \\
\hline $\begin{array}{l}\text { 2) Salutation entre chaque arrivant et tous les } \\
\text { membres déjà présents }\end{array}$ & \\
\hline $\begin{array}{l}\text { 3) Conversations informelles par petits groupes } \\
\text { de membres ayant choisi de s'asseoir à proximité }\end{array}$ & 5) et 6), plaisanteries \\
\hline $\begin{array}{l}\text { 4) Offre générale de nourriture (au minimum de la } \\
\text { cassave accompagnée de jus de manioc pimenté) }\end{array}$ & plaisanteries \\
\hline 5) Offre générale de coca & plaisanteries \\
\hline $\begin{array}{l}\text { 6) Partage des cigarettes (ciblé aux proches ou aux } \\
\text { hommes importants, voire à tous les fumeurs) }\end{array}$ & plaisanteries \\
\hline 7) Annonce des activités du jour par le capitaine & \\
\hline $\begin{array}{l}\text { 8) Activité A : discussion autour d'un thème } \\
\text { chamanique (si besoin) }\end{array}$ & 5) et 6) \\
\hline $\begin{array}{l}\text { 9) Activité B : Rituel chamanique (si besoin, mais } \\
\text { toujours au moins l'une des activités A ou B suivie } \\
\text { de l'annonce de ses résultats en langue indigène et } \\
\text { en espagnol) }\end{array}$ & 5) et 6) \\
\hline $\begin{array}{l}\text { 10) Annonce ou rappel des prochaines activités } \\
\text { concernant la communauté (travaux collectifs, } \\
\text { réunion, congrès, etc.) }\end{array}$ & 5) et 6) \\
\hline $\begin{array}{l}\text { 11) Annonce de la clôture de la réunion nocturne } \\
\text { par le capitaine. }\end{array}$ & 5) et 6) \\
\hline
\end{tabular}




\begin{tabular}{|l|l|}
\hline 12) Salutations finales entre chacun des membres & 5) et 6), plaisanteries \\
\hline 13) Départ des membres & \\
\hline
\end{tabular}

Comme je l'ai signalé plus haut, à ma connaissance, on ne rencontre à ces réunions que deux types d'activités ${ }^{27}: \mathrm{A}$ ) des discussions autour d'un thème chamanique, et $\mathrm{B}$ ) des rituels chamaniques.

Dans cet article, je ne traiterai pas des discussions à thème chamanique, car leur analyse requerrait l'exposé d'un nouvel extrait de transcriptions et par conséquent un nouvel article à part entière. Je me centrerai donc sur les rituels chamaniques.

Dans les réunions nocturnes du 15 octobre 2005 et du $1^{\text {er }}$ février 2008, j'ai pu relever ce qui est commun et ce qui est variable dans leur enchaînement. Parmi les variations possibles, je signalerai celles qui sont permises et celles qui ne le sont pas, d'après les réactions des participants ${ }^{28}$ dans le tableau suivant :

\begin{tabular}{|l|l|l|}
\hline \multicolumn{1}{|c|}{ Enchaînement fondamental } & Variations permises & $\begin{array}{c}\text { Variations } \\
\text { interdites }\end{array}$ \\
\hline $\begin{array}{l}\text { 1) Paroles cérémonielles } \\
\text { d'ouverture du rituel entre } \\
\text { guérisseurs }\end{array}$ & $\begin{array}{l}\text { Interruptions } \\
\text { quelconques }\end{array}$ \\
\hline $\begin{array}{l}\text { 2) Mise en accord entre } \\
\text { guérisseurs sur leur répartition } \\
\text { des tâches nécessaires au } \\
\text { traitement }\end{array}$ & $\begin{array}{l}\text { Interruptions } \\
\text { quelconques }\end{array}$ \\
\hline $\begin{array}{l}\text { 3) Préparation et regroupement } \\
\text { des éléments à traiter par un ou } \\
\text { plusieurs des guérisseurs }\end{array}$ & $\begin{array}{l}\text { Interruptions pertinentes } \\
\text { (récit d'un autre soigneur, } \\
\text { questions importantes, etc.) }\end{array}$ & $\begin{array}{l}\text { Interruptions } \\
\text { inopportunes } \\
\text { (d'enfants, par } \\
\text { exemple) }\end{array}$ \\
\hline
\end{tabular}

27. Par activité, j'entends un ensemble d'actes dont l'objectif est supposé avoir motivé l'organisation de l'assemblée.

28. Les réactions dont il faut le plus tenir compte sont évidemment celles des membres ayant la plus grande autorité au sein du rituel, c'est-à-dire les soigneurs et chamanes. 


\begin{tabular}{|l|l|l|}
\hline $\begin{array}{l}\text { 4) Mise à disposition de ces } \\
\text { éléments aux guérisseurs }\end{array}$ & Interruptions pertinentes & $\begin{array}{l}\text { Interruptions } \\
\text { inopportunes }\end{array}$ \\
\hline 5) Incantations des guérisseurs & Interruptions pertinentes & $\begin{array}{l}\text { Interruptions } \\
\text { inopportunes }\end{array}$ \\
\hline $\begin{array}{l}\text { Guérisseurs 1,2,..., } n: \\
\text { préalables à l'annonce des } \\
\text { résultats de l'incantation, }\end{array}$ & $\begin{array}{l}\text { Interruptions } \\
\text { quelconques }\end{array}$ \\
$\begin{array}{l}\text { 7) Annonce des résultats de } \\
\text { l'incantation, }\end{array}$ & $\begin{array}{l}\text { Questions de } \\
\text { l'interlocuteur principal }\end{array}$ & $\begin{array}{l}\text { Interruptions } \\
\text { inopportunes }\end{array}$ \\
$\begin{array}{l}\text { 8) Traduction en espagnol de } \\
\text { ces résultats }\end{array}$ & $\begin{array}{l}\text { Plaisanteries, rajouts par } \\
\text { le guérisseur d'annonces } \\
\text { oubliées ou non } \\
\text { mentionnées par lui ou le } \\
\text { traducteur }\end{array}$ & \\
\hline
\end{tabular}

Dès lors que l'on constate certaines variations à l'enchaînement commun, sans que ces variations n'entraînent de rabrouements, rappels à l'ordre ou disputes, je dirai que ces variations sont permises. Par exemple, peu avant l'extrait présenté ici, Milciades raconte un événement à Mario, alors que celui-ci avait déjà commencé son incantation. Dans le tableau ci-dessus, il est donc indiqué que durant les incantations des soigneurs (actes 5), on peut admettre les interruptions pertinentes.

De même durant la phase de traduction, il est permis de plaisanter (séquences 39-42, $45,47,139-140$ ) et les soigneurs peuvent également rajouter quelques dernières déclarations à traduire devant l'assemblée (séquences 173, 175).

Par contre, lors de l'annonce des résultats de l'incantation, chacun est censé observer le silence afin que toutes les personnes rassemblées puissent écouter. Et le soigneur est aussi en droit de faire patienter tout adulte cherchant à l'interrompre durant sa déclaration (séquence 85 ). Il devra néanmoins veiller à la bonne compréhension de son interlocuteur principal, par exemple, quand ce dernier lui demande de répéter ce qu'il n’a pas entendu (séquences 64, 110). 


\section{De l'incantation chamanique à l'interprétation en assemblée}

Avant d'examiner les cadres de référence mobilisés dans les échanges de paroles de notre extrait, il faut savoir que les compétences chamaniques des soigneurs fonctionnent principalement au moyen d'incantations murmurées dont les effets escomptés ont pour condition de fonctionnement le silence ou le secret (Fontaine, 2010, p. 10). Cela signifie qu'elles ne peuvent absolument pas être prononcées à voix haute au risque d'être écoutées, ni même expliquées en détail à des tiers. Il existe donc bel et bien une opposition entre les précautions de fonctionnement du chamanisme et les règles d'une assemblée communautaire. Tout rituel exercé au sein d'une réunion d'anciens doit alors adapter cette contradiction pour donner la juste mesure entre ce qu'il est possible de dévoiler et ce qui ne l'est pas, tout en donnant l'impression d'en avoir dit et fait suffisamment pour que l'assemblée soit globalement satisfaite de l'interprétation qui lui est donnée.

Commençons par étudier les incantations des soigneurs de langue yucuna ${ }^{29}$. L'incantation de Milciades est la plus courte et la plus communément employée. Elle a été faite avec de la coca, toujours utilisée comme moyen de divination et de protection à court terme (un ou deux mois), à la différence de la cire d'abeille qui, elle, est un moyen de divination à long terme (un ou deux ans), requérant une incantation beaucoup plus longue (parfois plus de 12 heures), difficile et contraignante sur le plan de la diète à respecter ${ }^{30}$. Entre les deux, l'incantation de Mario faite au genipa (lana maná) est utilisée pour la divination et la protection à court et moyen terme. Lors d'un travail de « maniement du monde » (eja'wá maná la'kana) à plusieurs soigneurs comme celui-ci, il est fréquent que l'on utilise les trois moyens pour se doter d'une protection plus fiable ainsi que d'une divination plus claire et complète.

Selon le moyen de traitement utilisé et les objectifs du jour, les incantations ont chacune leurs formules spécifiques que seuls les guérisseurs peuvent répéter, telles les invocations des entités que l'on cherche à contrôler ou les énumérations de lieux ou d'espèces (animales et végétales) nécessaires au traitement ${ }^{31}$.

29. Je me centrerai principalement sur celles de Milciades et Mario, tout en tenant compte également de celle d'Edilberto que j'ai également transcrite et traduite. Par contre, je laisse complètement de côté l'incantation miraña de Káiwa que je n'ai pas étudiée. Contrairement à celles des trois soigneurs de langue yucuna qui se sont beaucoup concertés tout au long du rituel, celle de Káiwa est restée discrète et indépendante.

30. C'est pourquoi Edilberto n'en assumera qu'une partie. La suite étant reprise par Mario. 31. Certains de ces matériaux ont fait l'objet de publications (FonTAIne, 2011, 2013b, 2014, 2015d). 
Le premier type de parole chamanique employé dans les incantations dont il est fait référence dans l'extrait est l'invocation. Elle est une partie fondamentale de l'incantation dans la mesure où c'est par elle que l'on espère manipuler ou rendre favorable toute entité dotée de pouvoirs intéressants. Pour mieux les saisir et les identifier, on cherche généralement à les caractériser précisément ; on les nomme par leurs différents noms en langue chamanique (lawichú chu) et on décrit souvent leurs caractéristiques corporelles.

Dans notre extrait, Milciades et Mario ont invoqué les Karipú Lakena, les quatre enfants créateurs du monde ${ }^{32}$ et le Jaguar Suprême (Yawi Chi'narikana), les divinités chamanes qui servent le plus souvent d'auxiliaires en matière de divination, et qui sont censées permettre aux initiés de ressentir des signalements dans leur corps ${ }^{33}$.

D'autres invocations sont souvent énumératives et concernent des espèces animales ou des groupes ethniques, qu'ils soient vus comme offensifs (séquences 3 , 12) ou comme victimes (séquences 6, 50, 57-62). Il convient alors de nommer tous leurs premiers ancêtres (nachi'narikana) sans en oublier ou déformer leurs noms (pour éviter de les vexer ou les mettre en colère), et de mentionner leurs terres d'origine (séquence 11).

Le moment le plus important est celui des interrogations qui, elles, sont toujours énumératives. Le soigneur nomme successivement chacune des éventualités nuisibles (séquence 10) en restant à l'affût du moment où l'une d'elles sera signalée par une sensation de son corps (kanakuwa). Lorsque l'un de ces dangers est ainsi signalé, le soigneur nomme toutes les ethnies qu'il connaît ${ }^{34}$ en attendant un nouveau signe de son corps pour savoir à laquelle appartiennent les personnes susceptibles d'être touchées (séquence 12).

Ainsi quand Milciades confie avoir « eu la forte sensation d'une arrivée de Blancs » (séquence 4), il dit ensuite avoir « énuméré toutes les éventualités pour

32. Plusieurs versions résumées ou extraites du mythe des Karipú Lakena ont été publiées : voir S. Schauer, J. Schauer, 1975 ; Jacopin, 1988 ; van der Hammen, 1991 ; SCHACKT, 1994 ; Fontaine, 2001, 2014. J'ai personnellement enregistré, transcrit, traduit et mis en ligne deux versions intégrales, l'une racontée par Milciades Yucuna, l'autre par Mario Matapi, voir FonTaine (2013c, 2015b).

33. Ces signalements sont particulièrement décrits dans le mythe des Karipú Lakena, notamment dans l'épisode du Yurupari. Ce sont eux qui auraient transmis cette faculté aux humains.

34. Dans ce genre d'énumération, on cherche souvent à préciser chaque genre d'entité en nommant son lieu d'origine (séq. 12). 
voir s'ils allaient nous faire du mal $\gg{ }^{35}$. L'absence de sensation corporelle durant l'énumération interrogative des dangers signifie qu'il n'y a rien à signaler (« ça devrait aller $\gg$, séquence 5).

Selon la même procédure que Milciades, Mario fait préalablement à son incantation du genipa une incantation à la coca (séquence 54) qui, lors de ses interrogations sur les éventualités nuisibles, le laisse ressentir des morsures de serpent $^{36}$ (séquence 56) atteignant les Tanimuca (séquences 62, 65) et les femmes yucuna (séquence 68), une grippe touchant enfants et adultes (séquences 55 , 71-78), des dangers en provenance des tapirs (séquences 82-83, 92-106) et des cerfs (séquences 86-90). Il rappelle qu'il a déjà annoncé cette même menace des tapirs aux Miraña, dont il a ressenti la « faute » envers eux (séquence 101), et qui pourrait avoir pour sanction la mort de la femme de Wasay, déjà très malade. Du point de vue de Mario, mais également de Milciades où de tout autre guérisseur de culture yucuna, ce genre de menace ne peut être écartée que par une incantation au genipa, qui avec une diète particulière permettrait à la vieille femme de disparaître de leur champ de perception, ou de ne plus être « exposée » (du terme yucuna meji'wá couramment employé pour dire « malade ») ${ }^{37}$.

De même que Milciades, Mario indique que des Blancs (de la guérilla) vont « surgir pour affronter les militaires » (séquences 108-109), et comme l'a déjà précisé Rey dans la traduction de l'annonce de son père (séquences $31-35)^{38}$, que cela se passera loin de la communauté, c'est-à-dire en forêt (séquences 112-113, 161-164).

Mario déclare ensuite ce qu'il a ressenti lors de son incantation du genipa (séquences 117-137). Toujours selon une même technique divinatoire, c'est par les sensations perçues au cours de ses interrogations qu'il fait ses prévisions, ici sur le moment le plus favorable de la prochaine beuverie dansante (à l'alcool d'ananas) permettant de clore le deuil (séquences 122-134).

Étant donné les circonstances dans lesquelles est décédé le dernier défunt, il faut savoir qu'une date trop avancée serait considérée comme risquée. Il s'agissait

35. On comprend alors qu'il parle de groupements armés venus d'autres régions. Il parle de la guérilla des Forces armées révolutionnaires de Colombie (FARC). En langue yucuna, on les appelle eja'wá chira'keño, « ceux qui se promènent en forêt ».

36. Dans ce cas, au moment où il nomme les serpents, le soigneur ressent physiquement une morsure au mollet ou à la cheville.

37. Voir Laurent Fontaine, 2010, annexe B, note 57.

38. Cette précision n'apparaît pas dans les dires de Milciades transcrits dans l'extrait. En fait, Milciades en avait déjà parlé avant à Mario. Rey fait donc la synthèse des deux annonces qu'il a entendues. 
de l'un des fils de la fille aînée de Milciades, âgé de 19 ans, s'étant suicidé par pendaison le 6 janvier dernier ${ }^{39}$. Selon la tradition, celui-ci avait été enterré dans la demeure de Milciades, à l'opposé de la pièce où dort ce dernier, entre deux pilotis de la maison auxquels plus personne n'installe son hamac pour dormir. S'agissant d'une mort violente, toute la famille de Milciades disait que son fantôme rôdait encore de nuit à proximité d'eux. On pouvait alors entendre les chiens aboyer, et certains prétendaient le voir ou l'entendre déambuler avec les bottes de son grand-père comme il aimait le faire de son vivant. Plusieurs adultes de la famille, particulièrement Rey, Edilberto et la femme de Milciades étaient soudainement tombés malades, ce que Milciades et d'autres soigneurs reliaient directement à l'intention du fantôme de les emporter avec lui.

Dans ce genre de mort tragique, le temps de deuil requiert au minimum trois mois, le temps que le fantôme du défunt s'apaise quelque peu en observant la tristesse et le regret qu'il suscite sur ses proches. Selon Edilberto, il serait fatal pour lui de chanter dans une cérémonie dansante avant la fin de ce laps de temps, car le fantôme lui-même ainsi que les esprits et les divinités faisant autorité sur le deuil et les cérémonies pourraient voir cela comme un manque de respect, une violation des interdits liés au deuil, et maudire toutes les personnes impliquées ${ }^{40}$. La préparation de la fin du deuil demande alors des compétences chamaniques assez importantes pour requérir l'aide d'un chamane comme Fermín (séquences 128131), particulièrement pour protéger contre les démons psychopompes la montée céleste de l'âme du défunt jusqu'à la maloca des Karipú Lakena ${ }^{41}$.

39. Milciades m'a raconté qu'il avait fait la prévision du suicide de son petit-fils, une dizaine de jours avant le drame, et qu'il l'avait supplié de ne pas en arriver à cet extrême. On dit que le jeune aurait fait un pacte avec le Diable, car il avait pris l'habitude d'en dessiner des portraits, et qu'il réussissait à se procurer facilement des sommes d'argent relativement importantes sans que personne ne sache comment. D'autres tentatives de suicide, dont l'une fut également fatale, ont aussi heurté le village depuis plusieurs mois. Certains soupçonnent une jeune fille carijona, venue avec sa famille de l'Araracuara, d'ensorceler ses courtisans, notamment en leur vendant de l'alcool d'ananas auquel elle ajouterait de son sang menstruel.

40. Il est d'ailleurs très mal vu pour les familles relativement proches des endeuillés d'organiser des bals, en sachant qu'ils ne peuvent les inviter. Une famille tanimuca de la communauté de Renacer (sur l'autre rive du Caqueta, face à la Pedrera) organisa ainsi un bal à la mi-février qui suscita l'indignation de Milciades, Edilberto et Mario.

41. Dans un long exposé transcrit et traduit par Fermín et sa femme, celui-ci m’a décrit l'ensemble de ce voyage des âmes défuntes. Arrivées chez les Karipú Lakena, ces derniers les jugeraient pour les renvoyer soit avec les autres âmes de leurs tribus soit chez des diablesses qui les brûlent et les renvoient parfois une dernière fois sur Terre sous forme de bourdon (Fontaine, 2015c). 
Étant donné le nombre d'obligations et d'interdits qui sont à respecter durant toute la durée du deuil, et non pas seulement lors de ces cérémonies, il est important de remarquer que le deuil a une influence qui peut englober tous les types de contexte de la vie courante (situations domestiques, visites, travaux collectifs, cérémonies) et qui, de ce fait, leur surajoutent leurs propres règles. De même pourrait-on dire de tous les autres moments rituels (yurupari, couvade, baptême, etc.).

Situé en plein dans ce champ d'influence du rituel, chaque mambeadero est ainsi un haut lieu d'application de ses règles. Mais à la différence des autres types de contexte, il est aussi un « espace public », ce qui le place également sous un second champ d'influence : celui des règles nationales et internationales. Deviennent alors prépondérants le devoir d'adapter les traditions pour ne pas entrer trop brusquement en opposition avec ces règles étrangères, et le droit de se faire comprendre et respecter en dehors de la situation même, y compris dans d'autres groupes ethniques et sociétés. C'est ainsi que l'espace public en tant qu'outil de communication et de diffusion proposé par les Blancs est adapté et réemployé de nos jours par les anciens de langue yucuna pour continuer à faire vivre leur culture.

\section{Bibliographie}

Benavides Martinez Jairo Norberto, 2007, «Palabra verdadera en los Uitotos para “vivir sabroso" », Poligrámas, n 28 , Bogota. http://poligramas.univalle. edu.co/28/9Jairo\%20Norberto\%20Benavidez.pdf [consulté le 20 mars 2015]

Fontaine Laurent, 2001, Paroles d'échange et Règles sociales chez les Indiens yucuna d'Amazonie colombienne, thèse de Doctorat, 2 tomes, université Sorbonne nouvelle - Paris 3. http://tel.archives-ouvertes.fr/tel-00596637 [consulté le 20 mars 2015]

FONTAINE Laurent, 2007, « Logiques modales et Anthropologie : des règles à la parole chez les Indiens yucuna d'Amazonie colombienne », l'Homme, no 184 , p. 131-154. http://www.cairn.info/revue-l-homme-2007-4-page-131.htm [consulté le 20 mars 2015] 
Fontaine Laurent, 2008, « les Nouvelles Interactions entre Yucuna et intervenants extérieurs (Colombie amazonienne) », Bulletin de la Sociétésuisse des Américanistes, n 70, p. 49-58. https://www.academia.edu/7797808/Les nouvelles_interactions_entre_Yucuna_et_intervenants_ext\%C3\%A9rieurs Colombie_amazonienne_-_In_Soci\%C3\%A9t\%C3\%A9 suisse_des Am\%C3\%A9ricanistes_2008_70_49-58 [consulté le 20 mars 2015]

Fontaine Laurent, 2010, « “Agents” ou “Patients” ? De l'agentivité des chamanes yucuna d'Amazonie colombienne », Ateliers du LESC, n 34, Nanterre. https://ateliers.revues.org/8526

Fontaine Laurent, 2011, « les Cours d'eau dans les incantations chamaniques des Indiens yucuna (Amazonie colombienne) », Journal de la Société des Américanistes, no 97-1, p. 119-149.

Fontaine Laurent, 2013a, « les Nouveaux Espaces publics chez les Yucuna d'Amazonie colombienne », Journal de la Société des Américanistes, n 99-1.

Fontaine Laurent, 2013b, « De l'agentivité mythique et incantatoire : le Mythe de Kawáirimi chez les Yucuna (Amazonie colombienne) », Ateliers d'anthropologie, $\mathrm{n}^{\circ}$ 39, Nanterre. http://ateliers.revues.org/9481

Fontaine Laurent, 2013c, Mythe des KaripúL Lakena, version de Milciades Yucuna. http://lacito.vjf.cnrs.fr/pangloss/tools/list_rsc.php?lg=Yucuna\&aff=yucuna [consulté le 12 juin 2014]

Fontaine Laurent, 2014, la Nuit pour apprendre: le chamanisme nocturne des Yucuna d'Amazonie colombienne, Nanterre : Société d'ethnologie, «Anthropologie de la nuit ».

FontaIne Laurent, 2015a, une Réunion nocturne chez Mario Matapi. http://site. laurentfontaine.free.fr/Films.html

Fontaine Laurent, 2015b, Mythe des Karipú Lakena, version de Mario Matapi. http://site.laurentfontaine.free.fr/Narrations.html

Fontaine Laurent, 2015c, le Chemin des âmes défuntes, version de Fermín (Je'rúriwa) Yucuna. http://site.laurentfontaine.free.fr/Savoirs_chamaniques. $\underline{\mathrm{html}}$ 
Fontaine Laurent, 2015d, Tropes et Agentivité dans les incantations des Yucuna d'Amazonie colombienne, ouvrage de recherche de HDR, 3 tomes, université Sorbonne nouvelle - Paris 3.

Jaco PIn Pierre-Yves, 1972, « Habitat et Territoire Yucuna », Journal de la Société des Américanistes, $\mathrm{n}^{\circ}$ 61-1, p. 107-139. http://www.persee.fr/web/revues/ home/prescript/article/jsa_0037-9174_1972_num_61_1_2115

Jacopin Pierre-Yves, 1981, la Parole générative de la mythologie des Indiens Yukuna, thèse de doctorat, université de Neuchâtel.

Jacopin Pierre-Yves, 1988, "On the Syntactic Structure of Myth, or the Yukuna Invention of Speech”, Cultural Anthropology, n 3-2, p. 131-158.

Jacopin Pierre-Yves, 2008, Paroles d'échange et Règles sociales chez les Indiens yucuna d'Amazonie colombienne, préf. de Laurent Fontaine, Paris : l'Harmattan, p. 5-10.

Parrado-Rosselli Angela, 2007, "A Collaborative Research Process Studying Fruit Availability and Seed Dispersal within an Indigenous Community in the Middle Caqueta River Region, Colombian Amazon”, Ecology and Society, vol. $12, \mathrm{n}^{\circ} 2$.

Schackт Jon, 1994, Nacimiento Yucuna. Reconstructive ethnography in Amazonia, thèse de doctorat, Université d'Oslo, 458 p.

Schauer Stanley, Schauer Junia, 1975, «Texto Yucuna por Quehuají Yucuna: la Historia de los Caripú Laquena», in Folclor indigena de Colombia, t. 1, Bogota, p. 252-333. http://www-01.sil.org/americas/colombia/pubs/ abstract.asp?id $=928474518846$

SERJE Margarita, 2003, « Malocas et Barracones : cas tradition, biodiversité et participation en Amazonie colombienne », Revue internationale des sciences sociales, $\mathrm{n}^{\circ} 178$.

Van Der Hammen Maria Clara, 1992, Elmanejo del mundo, Bogota: Tropenbos. 
72 Paroles publiques, paroles confidentielles $-\mathrm{n}^{\circ}$ 77-78

Résumé : El mambeadero est une situation de réunion nocturne dans laquelle les anciens mâchent la coca publiquement devant leur communauté pour réaliser des rituels ou pour être interrogés sur leurs savoirs. Les réunions nocturnes sont historiquement récentes chez les Yucuna puisqu'elles n'apparaissent qu'à la suite des premières réunions diurnes au milieu des années soixante-dix. Comme ces dernières, les réunions nocturnes s'organisent selon les mêmes règles obligeant à partager et à diffuser toutes les informations qui y sont énoncées. Mais dans les mambeaderos, les soigneurs et chamanes prononcent souvent des incantations et des diagnostics qui normalement devraient rester secrets et confidentiels. Par conséquent, les explications et les traductions qui sont fournies en public restent toujours assez superficielles. Un extrait de réunion nocturne transcrit et traduit des dialogues en yucuna et en espagnol est ici présenté et analysé pour examiner justement les décalages.

Mots clés : Amazonie, Yucuna, chamanisme, espace public, assemblée indigène, incantation, nuit

Abstract:El mambeadero is a nocturnal meeting, a situation where elders chew coca publicly in front of their community to conduct rituals or for being questioned about their knowledge. Nighttime meetings are historically recent among the Yucuna; they became organized after the first daytime meetings in the mid-seventies. Nocturnal meetings follow the same rules as daytime meetings; they require the sharing and dissemination of any information that is stated. But in mambeaderos, medicine men and shamans often pronounce incantations and diagnostics that normally should remain secret and confidential. Therefore, explanations and translations declared in public remain rather superficial. An extract of talk from a nocturnal meeting is transcribed and translated from the Yucuna; Spanish dialogues are presented and analyzed to examine precisely the discrepancies.

Keywords: Amazon, Yucuna, Shamanism, Public Space, Indigenous Assembly, Incantation, Night 